\title{
Heat and momentum transport in a multicomponent mixture far from equilibrium
}

\author{
V. Garzó* \\ Departamento de Fúsica, Universidad de Extremadura, \\ E-06071 Badajoz, Spain
}

(November 17, 2018)

\begin{abstract}
Explicit expressions for the heat and momentum fluxes are given for a low-density multicomponent mixture in a steady state with temperature and velocity gradients. The results are obtained from a formally exact solution of the Gross-Krook model [Phys. Rev. 102, 593 (1956)] of the Boltzmann equation for a multicomponent mixture. The transport coefficients (shear viscosity, viscometric functions, thermal conductivity and a cross coefficient measuring the heat flux orthogonal to the thermal gradient) are nonlinear functions of the velocity and temperature gradients and the parameters of the mixture (particle masses, concentrations, and force constants). The description applies for conditions arbitrarily far from equilibrium and is not restricted to any range of mass ratios, molar fractions and/or size ratios. The results show that, in general, the presence of the shear flow produces an inhibition in the transport of momentum and energy with respect to that of the Navier-Stokes regime. In the particular case of particles mechanically equivalent and in the tracer limit, previous results are recovered.

Keywords: Couette flow; Gross-Krook kinetic model; Multicomponent mixture; Nonlinear transport.

PACS number(s) : 51.10.+y, 05.20.Dd, 05.60.+w, 47.50.+d
\end{abstract}

\section{INTRODUCTION}

Needless to say, the study of transport phenomena in fluid mixtures is much more complicated than that of a single fluid. Not only is the number of transport coefficients much higher but also they depend on parameters such as the mass ratios, the molar fractions and/or the size ratios. These difficulties increase considerably when one attempts to analyze far from equilibrium states for which the linear relationships between fluxes and gradients do not apply. Due to the the complexity of the general problem, tractable specific situations must be considered. The study of such situations (for which a complete description can be given) is of great value since they allow us to gain some insight into the understanding of more general problems.

In order to capture the relevant aspects of nonlinear transport phenomena, a low-density $N$-component mixture with short-range interactions can be chosen as a prototype system. In this case, the essential information on the mixture is given by the one-particle velocity distribution functions $f_{i}(\mathbf{r}, \mathbf{v} ; t)(i=1, \ldots, N)$, which obey the set of coupled nonlinear Boltzmann equations [1]. For states near equilibrium, the constitutive Navier-Stokes equations for the heat and momentum fluxes (Fourier's heat law and Newton's viscosity law) can be derived, for instance, by using the Chapman-Enskog method [2]. Nevertheless, beyond the Navier-Stokes domain, the Chapman-Enskog calculations are prohibitively difficult and not applicable for large gradients. An interesting problem in which some insight into the mechanisms of heat and momentum transport far from equilibrium can be gained is that of steady planar Couette flow. The physical situation of the system is that of a multicomponent gas enclosed between two parallel plates in relative motion and maintained, in general, at different temperatures. These boundary conditions lead to temperature and density gradients coexisting with a velocity field. In the steady Couette flow there are two local parameters measuring the departure from equilibrium: the shear rate and the thermal gradient. Our aim is to get the relevant transport coefficients in terms of both gradients, as well as in terms of the parameters characterizing the mixture.

Unfortunatelly, due to the mathematical difficulties embodied in the nonlinear Couette flow problem, no analytic solution to the Boltzmann equation valid for arbitrary values of the shear rate and the thermal gradient is known, even for a single gas. Only in the limit of small shear rates, a perturbation solution in powers of the shear field through super-Burnett order has been recently worked out for a single gas of Maxwell molecules [3]. If one wants to obtain the full nonlinear dependence of the transport coefficients on the imposed gradients, either one performs computer simulations or on the analytical side one considers kinetic models or the Grad method $\|$. Here, we take the second

*Electronic address: vicenteg@unex.es 
route and consider a kinetic model. Specifically, we use the nonlinear kinetic model for mixtures proposed by Gross and Krook (GK) [5]. This model is constructed in the same spirit as the well-known Bhatnagar-Gross-Krook (BGK) model of a single gas [6], which admits an exact solution for the steady nonlinear Couette flow [7,8]. The GK model preserves the most important physical properties of the true Boltzmann equation while allowing for more complete calculations.

As mentioned above, the goal of this paper is to obtain exactly the pressure tensor (momentum transport) and the heat flux (energy transport) of a multicomponent mixture under Couette flow in the context of the GK model. We assume that the molar fractions are constant, so that no mutual diffusion appears in the system. On the other hand, no restriction on the masses, concentrations, and force constants will be considered. Consequently, the transport coefficients are nonlinear functions of both the shear rate and the thermal gradient and of the parameters of the mixture. Obviously, our results reduce to those obtained from the BGK model in the case of particles mechanically equivalent [7]. Further, when one considers a binary mixture and takes the tracer limit (molar fraction of one of the components much smaller than 1), previous results derived from the GK model are also recovered [9].

The plan of the paper is as follows. Section II starts with a brief survey of the GK model for an $N$-component mixture. Then, the nolinear Couette flow is introduced. In the same way as in the single gas case, we propose a solution characterized by constant pressure and linear velocity and parabolic temperature profiles with respect to a scaled variable. The consistency of the assumed profiles is fulfilled in Sec. III from a formal solution of the GK model. The consistency condition for the temperature yields a system of $N$ implicit equations which give the shear rate dependence of the partial temperatures $T_{i}$ of each species. For nonzero shear rates, the $T_{i}$ 's are different, so that the kinetic energy is not equally distributed among the different species. This effect is generic for multicomponent systems in far from equilibrium states and is consistent with other studies of mixtures out of equilibrium 10 12]. Apart from the partial temperatures, the pressure tensor and the heat flux vector are also explicitly determined. From these fluxes the main transport coefficients of the problem can be identified. Specifically, there are five relevant transport coefficients: the generalized shear viscosity function, two viscometric functions, the generalized thermal conductivity function, and a coefficient measuring cross effects in the heat flux (heat flux along the $x$ direction due to a thermal gradient along the $y$ direction). The expressions of these functions are written in Sec. IV. The particular case of a binary mixture $(N=2)$ is considered in Sec. $\mathrm{V}$, where the shear rate dependence of the above transport coefficients is illustrated for several values of the parameters of the mixture. Finally, we close the paper in Sec. VI with a brief discussion.

\section{THE KINETIC MODEL AND THE PROBLEM}

We consider an $N$-component mixture. In the low-density regime, the one-particle velocity distribution function $f_{i}(\mathbf{r}, \mathbf{v} ; t)$ of species $i(i=1, \ldots, N)$ obeys the Boltzmann equation [2]

$$
\frac{\partial}{\partial t} f_{i}+\mathbf{v} \cdot \nabla f_{i}=K_{i i}\left[f_{i}, f_{i}\right]+\sum_{j \neq i} K_{i j}\left[f_{i}, f_{j}\right]
$$

where $K_{i j}\left[f_{i}, f_{j}\right]$ is the nonlinear Boltzmann collision operator. The first and second terms on the right-hand side represent self- and cross-collisions, respectively. They conserve the number of particles of each species, the total momentum and the total energy. The local number density $n_{i}$ and mean velocity $\mathbf{u}_{i}$ of species $i$ are defined as

$$
\begin{gathered}
n_{i}=\int d \mathbf{v} f_{i}, \\
\mathbf{u}_{i}=\frac{1}{n_{i}} \int d \mathbf{v} \mathbf{v} f_{i} .
\end{gathered}
$$

These quantities define the total number density $n=\sum_{i} n_{i}$ and the flow velocity

$$
\mathbf{u}=\frac{1}{\rho} \sum_{i=1}^{N} \rho_{i} \mathbf{u}_{i}
$$

where $\rho_{i}=m_{i} n_{i}$ and $\rho=\sum_{i} \rho_{i}, m_{i}$ being the mass of a particle of species $i$. It is also convenient to define a temperature $T_{i}$ for each species through 


$$
\frac{3}{2} n_{i} k_{B} T_{i}=\frac{m_{i}}{2} \int d \mathbf{v}\left(\mathbf{v}-\mathbf{u}_{i}\right)^{2} f_{i},
$$

which is a measure of its mean kinetic energy per particle. Here, $k_{B}$ is the Boltzmann constant. From these partial temperatures, the temperature $T$ of the mixture (which is the relevant one at a hydrodynamic level) is given by

$$
n k_{B} T=\sum_{i=1}^{N}\left[n_{i} k_{B} T_{i}+\frac{1}{3} \rho_{i}\left(\mathbf{u}_{i}-\mathbf{u}\right)^{2}\right] .
$$

The corresponding balance equations associated with $n_{i}, \mathbf{u}$ and $T$ can be easily obtained from the set of Boltzmann equations (1):

$$
\begin{gathered}
D_{t} n_{i}+n_{i} \nabla \cdot \mathbf{u}+\frac{\nabla \cdot \mathbf{J}_{i}}{m_{i}}=0, \\
D_{t} \mathbf{u}+\rho^{-1} \nabla \mathrm{P}=0, \\
D_{t} T-\frac{T}{n} \sum_{i} \frac{\nabla \cdot \mathbf{J}_{i}}{m_{i}}+\frac{2}{3 n k_{B} T}(\nabla \cdot \mathbf{q}+\mathrm{P}: \nabla \mathbf{u})=0 .
\end{gathered}
$$

In the above equations, $D_{t} \equiv \partial_{t}+\mathbf{u} \cdot \nabla$, and we have introduced the dissipative fluxes of mass

$$
\mathbf{J}_{i}=m_{i} \int d \mathbf{v} \mathbf{V} f_{i},
$$

momentum (pressure tensor)

$$
\mathrm{P}=\sum_{i=1}^{N} m_{i} \int d \mathbf{V} \mathbf{V} \mathbf{V} f_{i}=\sum_{i=1}^{N} \mathrm{P}_{i},
$$

and energy (heat flux)

$$
\mathbf{q}=\sum_{i=1}^{N} \frac{m_{i}}{2} \int d \mathbf{v} V^{2} \mathbf{V} f_{i}=\sum_{i=1}^{N} \mathbf{q}_{i},
$$

where $\mathbf{V}=\mathbf{v}-\mathbf{u}$ is the peculiar velocity. These fluxes define the relevant transport coefficients of the mixture.

In general, the set of $N$ nonlinear Boltzmann equations (1) cannot be solved, especially in far from equilibrium situations. This is basically due to the complex mathematical structure of the Boltzmann collision operators. In order to overcome such difficulties one can resort to kinetic models in which case the Boltzmann collision operators are replaced by simpler terms that preserve their main physical properties. Here we consider the well-known Gross-Krook (GK) model [5], where the Boltzmann terms $K_{i j}\left[f_{i}, f_{j}\right]$ in Eqs. (11) are replaced by simple relaxation terms of the form

$$
K_{i j}^{\mathrm{GK}}=-\nu_{i j}\left(f_{i}-f_{i j}\right),
$$

where $\nu_{i j}$ is an effective collision frequency and the reference distribution functions $f_{i j}$ are given by

$$
f_{i j}=n_{i}\left(\frac{m_{i}}{2 \pi k_{B} T_{i j}}\right)^{3 / 2} \exp \left[-\frac{m_{i}}{2 k_{B} T_{i j}}\left(\mathbf{v}-\mathbf{u}_{i j}\right)^{2}\right] .
$$

Here, we have introduced the fields

$$
\begin{gathered}
\mathbf{u}_{i j}=\frac{m_{i} \mathbf{u}_{i}+m_{j} \mathbf{u}_{j}}{m_{i}+m_{j}} \\
T_{i j}=T_{i}+2 \frac{m_{i} m_{j}}{\left(m_{i}+m_{j}\right)^{2}}\left[\left(T_{j}-T_{i}\right)+\frac{m_{i}}{6 k_{B}}\left(\mathbf{u}_{i}-\mathbf{u}_{j}\right)^{2}\right] .
\end{gathered}
$$


The above expressions are obtained by requiring that momentum and energy moments of $K_{i j}^{\mathrm{GK}}$ be the same as those of the Boltzmann operator for Maxwell molecules (i.e., particles interacting through a potential of the form $\left.\Phi_{i j}=\kappa_{i j} r^{-4}\right)$. This allows one to identify $\nu_{i j}$ as [5]

$$
\nu_{i j}=A n_{j}\left[\kappa_{i j} \frac{m_{i}+m_{j}}{m_{i} m_{j}}\right]^{1 / 2},
$$

where $A=4 \pi \times 0.422$. Although we are considering Maxwell molecules, we can assign an effective diameter $\sigma_{i j}$ to the interaction between particles of species $i$ and $j$. Dimensional analysis allows one to interpret the ratio $\left(\kappa_{i j} / \kappa_{j j}\right)^{1 / 4}$ as the size ratio $\sigma_{i j} / \sigma_{j j}$. On the other hand, it must be remarked that the results derived in this paper could in principle be extended to more general potentials.

We now describe the problem we are interested in. Let us assume that the $N$-component mixture is enclosed between two parallel plates in relative motion and kept at different temperatures. Let the $x$-axis be parallel to the direction of motion and the $y$-axis be normal to the plates. We want to analyze a steady state with velocity $\left(u_{x}\right)$ and temperature $(T)$ gradients along the $y$ direction (steady Couette flow). These boundary conditions give rise to heat and momentum transport, the relevant fluxes being the pressure tensor and the heat flux. Our goal is to get these transport properties in terms of the imposed gradients and the parameters of the mixture, namely, masses, concentrations, and "sizes" of the particles. In the geometry of the steady Couette flow and in the context of the GK model, the set of $N$ coupled equations for the mixture reads

$$
\begin{aligned}
v_{y} \frac{\partial}{\partial y} f_{i} & =-\nu_{i i}\left(f_{i}-f_{i i}\right)-\sum_{j \neq i} \nu_{i j}\left(f_{i}-f_{i j}\right) \\
& =-\nu_{i} f_{i}+\sum_{j=1}^{N} \nu_{i j} f_{i j}
\end{aligned}
$$

where

$$
\nu_{i}=\sum_{j=1}^{N} \nu_{i j}=\nu_{i i}+\sum_{j \neq i} \nu_{i j}
$$

is the total collision frequency for a particle of species $i$. In the same way as in previous works, here we are interested in studying the properties of the mixture in the bulk region far away from the plates. Thus, instead of introducing the appropriate boundary conditions for Eqs. (18), we guess the spatial dependence of the hydrodynamic fields and then we verify their consistency. In this sense, we expect to describe transport phenomena in the bulk of the system by looking for a consistent solution regardless of the actual features of the boundaries.

In the case of all the species being mechanically identical, $\mathbf{u}_{i}=\mathbf{u}, T_{i}=T$ and the GK model reduces to the well-known (closed) BGK equation [6] for the total distribution function $f=\sum_{i} f_{i}$. In this case, the BGK model admits a consistent solution characterized by a constant pressure, and linear velocity $u_{x}$ and parabolic temperature $T$ profiles with respect to a scaled variable [7, 8 . This variable takes into account the fact that the rate at which collisions take place is nonuniform. The above solution is not restricted to small velocity and temperature gradients and thus provides a rare detailed description of nonlinear heat and momentum transport far from equilibrium. Guided by these results for the one-component case, a similar solution is proposed here for the mixture. First, on physical grounds one expects that the steady Couette flow for the mixture is characterized by (a) constant molar fractions $x_{i}=n_{i} / n,(\mathrm{~b})$ the absence of mutual diffusion, i.e., $\mathbf{u}_{i}=\mathbf{u}$, and (c) constant temperature ratios $\chi_{i} \equiv T_{i} / T$. In addition, we assume that the partial pressure $p_{i}=n_{i} k_{B} T_{i}$, the velocity $u_{i, x}$, and the partial temperature $T_{i}$ have the forms:

$$
\begin{gathered}
p_{i} \equiv n_{i} k_{B} T_{i}=\text { const } \\
\frac{1}{\nu_{i}(y)} \frac{\partial}{\partial y} u_{i, x}=a_{i}=\text { const } \\
{\left[\frac{1}{\nu_{i}(y)} \frac{\partial}{\partial y}\right]^{2} T_{i}=-\frac{2 m_{i}}{k_{B}} \gamma_{i}\left(a_{i}\right)=\text { const }}
\end{gathered}
$$

The dimensionless functions $\gamma_{i}$ must be explicitly determined by consistency. The conditions $\mathbf{u}_{i}=\mathbf{u}$ and $\chi_{i}=$ const imply, respectively, that 


$$
\begin{aligned}
\nu_{i} a_{i} & =\nu_{j} a_{j}, \\
\frac{m_{i} \gamma_{i} \nu_{i}^{2}}{\chi_{i}} & =\frac{m_{j} \gamma_{j} \nu_{j}^{2}}{\chi_{j}},
\end{aligned}
$$

where use has been made of the fact that the ratios $\nu_{i} / \nu_{j}$ are constant. According to Eqs. (23) and (24), only one of the shear rates $a_{i}$ and only one of the parameters $\gamma_{i}(i=1, \ldots, N)$ is independent. The temperature ratios $\chi_{i}$ depend on the shear rate and on the parameters of the mixture. Obviously, $\chi_{i}=1$ if (i) the mixture is at equilibrium or (ii) the particles are mechanically equivalent $\left(m_{i}=m\right.$ and $\left.\kappa_{i j}=\kappa\right)$. The explicit calculation of the temperature ratios is one of the main objectives of this paper.

To verify the consistency of the assumed solution it is necessary to prove that

$$
\int d \mathbf{v}\left\{1, \mathbf{V}, V^{2}\right\} f_{i}=\left\{n_{i}, 0,3 \frac{p_{i}}{m_{i}}\right\}
$$

The fulfillment of these conditions will be carried out in the next Section.

\section{CONSISTENCY OF THE SOLUTION. CALCULATION OF THE FLUXES}

In this Section we show that the relations (25) are verified and obtain the pressure tensor and the heat fux. To do that, let us consider the formal solution to Eq. (18) given by

$$
f_{i}=\left(1+\frac{v_{y}}{\nu_{i}} \frac{\partial}{\partial y}\right)^{-1} \sum_{j=1}^{N} \frac{\nu_{i j}}{\nu_{i}} f_{i j}
$$

Notice that when the operator $\left[1+\left(v_{y} / \nu_{i}\right)(\partial / \partial y)\right]^{-1}$ acts on the hydrodynamic quantities the expressions (20)-(22) are assumed to hold. By using this formal solution in the Appendix we show that the conditions for the density and the flow velocity are satisfied with independence of the value of the parameters $\gamma_{i}$ in Eq. (22) for the temperature $T_{i}(y)$. A similar analysis for the temperature condition shows that a solution exists only for a particular choice of $\gamma_{i}$ given implicitly by the equation:

$$
\frac{3}{2} \sum_{j \neq i}\left(1-\chi_{i j}\right) \nu_{i j}=\sum_{j=1}^{N} \nu_{i j} \chi_{i j}\left\{a_{i}^{2} F_{1}\left(\gamma_{i j}\right)-\gamma_{i j}\left[2 F_{2}\left(\gamma_{i j}\right)+3 F_{1}\left(\gamma_{i j}\right)\right]\right\}
$$

Here, we have introduced the quantities $\gamma_{i j}=\chi_{i j} \gamma_{i}$,

$$
\chi_{i j}=1+2 \frac{m_{i} m_{j}}{\left(m_{i}+m_{j}\right)^{2}}\left(\frac{\chi_{j}}{\chi_{i}}-1\right) .
$$

In addition, the functions $F_{r}\left(\gamma_{i j}\right)$ are defined as

$$
F_{r}\left(\gamma_{i j}\right)=\left(\frac{d}{d \gamma_{i j}} \gamma_{i j}\right)^{r} F_{0}\left(\gamma_{i j}\right)
$$

with

$$
F_{0}\left(\gamma_{i j}\right)=\frac{2}{\gamma_{i j}} \int_{0}^{\infty} d t t e^{-t^{2} / 2} K_{0}\left(2 \gamma_{i j}^{-1 / 4} t^{1 / 2}\right),
$$

$K_{0}$ being the zeroth-order modified Bessel function [13. The set of $N$ coupled equations (27) must be numerically solved subject to the constraints (24) and

$$
\sum_{i=1}^{N} x_{i} \chi_{i}=1
$$


From a mathematical point of view, the problem is now well posed since we have $N$ independent equations (27) for a set of $N$ unknowns, say for instance $\left\{\gamma_{1}, \chi_{1}, \chi_{2}, \cdots, \chi_{N-1}\right\}$. The solution of these $N$ implicit equations gives the above quantities as functions of the shear rate and the parameters of the mixture (masses, concentrations, and sizes). This completes the confirmation of the consistency conditions for the hydrodynamic fields.

Once the functions $\gamma_{i}$ and $\chi_{i}$ are known, one can derive explicit expressions for the fluxes. In the case of the Couette flow, the mass flux $\mathbf{J}_{i}$ vanishes, and so the relevant fluxes are the pressure tensor (11) and the heat flux (12). We begin with the nonzero elements of the pressure tensor. In the Appendix it is shown that they can be written as

$$
\begin{gathered}
P_{x x}=n k_{B} T \sum_{i=1}^{N} \frac{x_{i} \chi_{i}}{\nu_{i}} \sum_{j=1}^{N} \nu_{i j} \chi_{i j}\left\{1+4 \gamma_{i j}\left[F_{1}\left(\gamma_{i j}\right)+F_{2}\left(\gamma_{i j}\right)\right]\right\}, \\
P_{y y}=n k_{B} T \sum_{i=1}^{N} \frac{x_{i} \chi_{i}}{\nu_{i}} \sum_{j=1}^{N} \nu_{i j} \chi_{i j}\left\{1-2 \gamma_{i j}\left[F_{1}\left(\gamma_{i j}\right)+2 F_{2}\left(\gamma_{i j}\right)\right]\right\}, \\
P_{z z}=n k_{B} T \sum_{i=1}^{N} \frac{x_{i} \chi_{i}}{\nu_{i}} \sum_{j=1}^{N} \nu_{i j} \chi_{i j}\left[1-2 \gamma_{i j} F_{1}\left(\gamma_{i j}\right)\right], \\
P_{x y}=-n k_{B} T \sum_{i=1}^{N} \frac{x_{i} \chi_{i}}{\nu_{i}^{2}} \sum_{j=1}^{N} \nu_{i j} \chi_{i j} F_{0}\left(\gamma_{i j}\right) \frac{\partial u_{x}}{\partial y} .
\end{gathered}
$$

Notice that the pressure tensor does not depend on the thermal gradient. With respect to the heat flux, its nonzero components are $q_{x}$ and $q_{y}$. The fact that $q_{x} \neq 0$ is a direct consequence of the presence of the shear flow. These components are also evaluated in the Appendix and the result is

$$
\begin{aligned}
q_{y}= & -\frac{5}{2} n k_{B}^{2} T \sum_{i=1}^{N} \frac{x_{i} \chi_{i}^{2}}{5 m_{i} \nu_{i}^{2}} \sum_{j=1}^{N} \nu_{i j} \chi_{i j}\left\{3 F_{1}\left(\gamma_{i j}\right)+2 F_{2}\left(\gamma_{i j}\right)\right. \\
& \left.+2 a_{i}^{2}\left[4 F_{4}\left(\gamma_{i j}\right)+4 F_{3}\left(\gamma_{i j}\right)+F_{2}\left(\gamma_{i j}\right)\right]\right\} \frac{\partial T}{\partial y}, \\
q_{x}= & n k_{B}^{2} T \sum_{i=1}^{N} a_{i} \frac{x_{i} \chi_{i}^{2}}{m_{i} \nu_{i}^{2}} \sum_{j=1}^{N} \nu_{i j} \chi_{i j}\left\{5 F_{2}\left(\gamma_{i j}\right)+2 F_{3}\left(\gamma_{i j}\right)\right. \\
+ & \left.2 a_{i}^{2}\left[4 F_{5}\left(\gamma_{i j}\right)+8 F_{4}\left(\gamma_{i j}\right)+5 F_{3}\left(\gamma_{i j}\right)+F_{2}\left(\gamma_{i j}\right)\right]\right\} \frac{\partial T}{\partial y} .
\end{aligned}
$$

From Eqs. (35) and (36), it is easy to prove the identity $\partial_{y} q_{y}=-P_{x y} \partial_{y} u_{x}$, which is in fact required by the energy conservation equation (9) to support the forms for the hydrodynamic profiles.

\section{GENERALIZED TRANSPORT COEFFICIENTS}

It is useful to summarize the above results for the fluxes in terms of appropriate scalar transport coefficients. To do that, let us reduce the velocity gradient $\partial_{y} u_{x}$ with respect to an effective collision frequency $\nu$. Since our description applies for arbitrary mass, concentration and size ratios, we choose for simplicity the average collision frequency

$$
\nu=\frac{1}{N(N-1)} \sum_{i=1}^{N} \sum_{j \neq i} \frac{n}{n_{j}} \nu_{i j}
$$

and define the (dimensionless) shear rate

$$
a=\frac{1}{\nu} \frac{\partial}{\partial y} u_{x}=\text { const }
$$


as the relevant nonequilibrium parameter. Obviously, $a_{i}=\left(\nu / \nu_{i}\right) a$.

Momentum transport is typically characterized by three rheological functions. The $x y$ element for shear stressess can be written in the form of a generalized Newton's viscosity law

$$
P_{x y}=-\eta_{0} F_{\eta}(a) \frac{\partial u_{x}}{\partial y}
$$

where

$$
\eta_{0}=n k_{B} T \sum_{i=1}^{N} \frac{x_{i}}{\nu_{i}}
$$

is the Navier-Stokes shear viscosity of the mixture [5] and the explicit expression of $F_{\eta}(a)$ can be obtained from Eq. (35) as

$$
F_{\eta}(a)=\left(\sum_{i=1}^{N} \frac{x_{i}}{\nu_{i}}\right)^{-1} \sum_{i=1}^{N} \frac{x_{i} \chi_{i}}{\nu_{i}^{2}} \sum_{j=1}^{N} \nu_{i j} \chi_{i j} F_{0}\left(\gamma_{i j}\right) .
$$

When $a=0, F_{\eta}=1$, so that this function describes the nonlinear rheological effects associated with the transport of the $x$ component of the momentum along the $y$ direction. Normal stresses are measured by the dimensionless viscometric functions $\Psi_{1,2}(a)$ :

$$
\begin{aligned}
& \Psi_{1}(a)=\frac{P_{y y}-P_{x x}}{p a^{2}}, \\
& \Psi_{2}(a)=\frac{P_{z z}-P_{y y}}{p a^{2}},
\end{aligned}
$$

where $p=n k_{B} T$. The explicit expressions for the viscometric functions can be easily obtained by substituting Eqs. (32)-(34) into Eqs. (43) and (44). The result is

$$
\begin{gathered}
\Psi_{1}(a)=-\frac{2}{a^{2}} \sum_{i=1}^{N} \frac{x_{i} \chi_{i}}{\nu_{i}} \sum_{j=1}^{N} \nu_{i j} \chi_{i j} \gamma_{i j}\left[3 F_{1}\left(\gamma_{i j}\right)+4 F_{2}\left(\gamma_{i j}\right)\right] \\
\Psi_{2}(a)=\frac{4}{a^{2}} \sum_{i=1}^{N} \frac{x_{i} \chi_{i}}{\nu_{i}} \sum_{j=1}^{N} \nu_{i j} \chi_{i j} \gamma_{i j} F_{2}\left(\gamma_{i j}\right) .
\end{gathered}
$$

The heat flux introduces two relevant transport coefficients, $F_{\lambda}$ and $\Phi$, defined respectively as

$$
\begin{aligned}
& q_{y}=-\lambda_{0} F_{\lambda}(a) \frac{\partial T}{\partial y}, \\
& q_{x}=-\lambda_{0} \Phi(a) a \frac{\partial T}{\partial y} .
\end{aligned}
$$

Here, $\lambda_{0}$ is the Navier-Stokes thermal conductivity coefficient of the mixture given by [5]

$$
\lambda_{0}=\frac{5}{2} p k_{B} \sum_{i=1}^{N} \frac{x_{i}}{m_{i} \nu_{i}} .
$$

Equation (47) can be interpreted as a generalization of Fourier's law with the thermal conductivity modified by the rheological quantity $F_{\lambda}(a)$, a nonlinear function of the shear rate and the parameters of the mixture. Equation (48) provides information on the anisotropy induced by the Couette flow in the transport of energy. It gives a heat flux along the $x$ axis due to a thermal gradient along the $y$ axis. This effect is absent in the Navier-Stokes regime, being 
of first order in both the shear rate and the temperature gradient. Additional effects are described by the function $\Phi(a)$. The explicit expressions of $F_{\lambda}$ and $\Phi$ can be derived from Eqs. (36) and (37) as

$$
\begin{aligned}
F_{\lambda}(a)= & \left(\sum_{i=1}^{N} \frac{x_{i}}{m_{i} \nu_{i}}\right)^{-1} \sum_{i=1}^{N} \frac{x_{i} \chi_{i}^{2}}{5 m_{i} \nu_{i}^{2}} \sum_{j=1}^{N} \nu_{i j} \chi_{i j}\left\{3 F_{1}\left(\gamma_{i j}\right)+2 F_{2}\left(\gamma_{i j}\right)\right. \\
& \left.+2 \frac{\nu^{2} a^{2}}{\nu_{i}^{2}}\left[4 F_{4}\left(\gamma_{i j}\right)+4 F_{3}\left(\gamma_{i j}\right)+F_{2}\left(\gamma_{i j}\right)\right]\right\}, \\
\Phi(a)=- & \frac{2}{5} \nu\left(\sum_{i=1}^{N} \frac{x_{i}}{m_{i} \nu_{i}}\right)^{-1} \sum_{i=1}^{N} \frac{x_{i} \chi_{i}^{2}}{m_{i} \nu_{i}^{3}} \sum_{j=1}^{N} \nu_{i j} \chi_{i j}\left\{5 F_{2}\left(\gamma_{i j}\right)+2 F_{3}\left(\gamma_{i j}\right)\right. \\
& \left.+2 \frac{\nu^{2} a^{2}}{\nu_{i}^{2}}\left[4 F_{5}\left(\gamma_{i j}\right)+8 F_{4}\left(\gamma_{i j}\right)+5 F_{3}\left(\gamma_{i j}\right)+F_{2}\left(\gamma_{i j}\right)\right]\right\} .
\end{aligned}
$$

Before closing this Section, let us explicitly write the corresponding expressions of the generalized transport coefficients in the simple case of particles mechanically equivalent. In this limit, $m_{i}=m, \kappa_{i j}=\kappa, \nu=A n(2 \kappa / m)^{1 / 2}$, $\gamma_{i}=\gamma$, and $\chi_{i}=1$. Consequently, Eqs. (42), (45), (46), (50), and (51) reduce, respectively, to

$$
\begin{gathered}
F_{\eta}(a)=F_{0}(\gamma), \\
\Psi_{1}(a)=-\frac{2}{a^{2}} \gamma\left[3 F_{1}(\gamma)+4 F_{2}(\gamma)\right] \\
\Psi_{2}(a)=\frac{4}{a^{2}} \gamma F_{2}(\gamma), \\
F_{\lambda}(a)=\frac{1}{5}\left\{3 F_{1}(\gamma)+2 F_{2}(\gamma)+2 a^{2}\left[4 F_{4}(\gamma)+4 F_{3}(\gamma)+F_{2}(\gamma)\right]\right\} \\
\Phi(a)=-\frac{2}{5}\left\{5 F_{2}(\gamma)+2 F_{3}(\gamma)+2 a^{2}\left[4 F_{5}(\gamma)+8 F_{4}(\gamma)+5 F_{3}(\gamma)+F_{2}(\gamma)\right]\right\}
\end{gathered}
$$

All these expressions coincide with those previously derived from the BGK equation [7,14].

\section{AN ILLUSTRATIVE EXAMPLE: A BINARY MIXTURE}

Thus far, all the results hold for an $N$-component mixture. For the sake of illustration, in this Section we will consider the case of a binary mixture $(N=2)$. Therefore, the corresponding set of two implicit equations $(27)$ give $\gamma_{1}$ and $\chi_{1}$ as functions of $a$ and the parameters of the mixture, namely, the mass ratio $\mu \equiv m_{1} / m_{2}$, the concentration ratio $\delta \equiv x_{1} / x_{2}$, and the size ratios $w_{11} \equiv \kappa_{11} / \kappa_{12}$, and $w_{22} \equiv \kappa_{22} / \kappa_{12}$. Although the natural nonequilibrium parameter is the shear rate $a$, from a practical point of view it is convenient to take $\gamma_{1}$ as an independent variable and express all the unknowns in terms of this parameter. In this sense, $\chi_{1}$ is defined by the implicit equation

$$
\left(\frac{\nu_{1}}{\nu_{2}}\right)^{2} \frac{\sum_{i=1}^{2} \nu_{1 i} \chi_{1 i} F_{1}\left(\gamma_{1 i}\right)}{\sum_{i=1}^{2} \nu_{2 i} \chi_{2 i} F_{1}\left(\gamma_{2 i}\right)}=\frac{3 \nu_{12}\left(1-\chi_{12}\right)+2 \sum_{i=1}^{2} \nu_{1 i} \chi_{1 i} \gamma_{1 i}\left[2 F_{2}\left(\gamma_{1 i}\right)+3 F_{1}\left(\gamma_{1 i}\right)\right]}{3 \nu_{21}\left(1-\chi_{21}\right)+2 \sum_{i=1}^{2} \nu_{2 i} \chi_{2 i} \gamma_{2 i}\left[2 F_{2}\left(\gamma_{2 i}\right)+3 F_{1}\left(\gamma_{2 i}\right)\right]},
$$

and the shear rate $a$ is given by

$$
a^{2}=\frac{1}{2}\left(\frac{\nu_{1}}{\nu}\right)^{2} \frac{3 \nu_{12}\left(1-\chi_{12}\right)+2 \sum_{i=1}^{2} \nu_{1 i} \chi_{1 i} \gamma_{1 i}\left[2 F_{2}\left(\gamma_{1 i}\right)+3 F_{1}\left(\gamma_{1 i}\right)\right]}{\sum_{i=1}^{2} \nu_{1 i} \chi_{1 i} F_{1}\left(\gamma_{1 i}\right)} .
$$

In the above equations, $\gamma_{2}$ and $\chi_{2}$ are related to $\gamma_{1}$ and $\chi_{1}$ through Eqs. (24) and (31). In general, Eq. (57) must be solved numerically. However, $\gamma_{1}$ and $\chi_{1}$ can be explicitly derived in some limiting cases. For instance, in the limit of small shear rates $\gamma_{1} \rightarrow \gamma_{1}^{(0)} a^{2}$, and $\chi_{1} \rightarrow 1+\chi_{1}^{(0)} a^{2}$, where 


$$
\begin{gathered}
\gamma_{1}^{(0)}=\frac{1}{5}\left(\frac{\nu}{\nu_{1}}\right)^{2} \frac{\nu_{1}+\delta \nu_{2}}{\mu \nu_{1}+\delta \nu_{2}} \\
\chi_{1}^{(0)}=\frac{1}{3} \frac{\left(\mu^{2}-1\right)(1+\mu)(1+\delta)}{\mu} \frac{\nu_{12}}{\mu \nu_{1}+\delta \nu_{2}} .
\end{gathered}
$$

The temperature ratio $T_{1} / T_{2}$ is a measure of the lack of equidistribution of the kinetic energy. This quantity is plotted in Fig. 1 as a function of the shear rate for $w_{11}=w_{22}=1$, a concentration ratio $\delta=2$ and two different values of the mass ratio: $\mu=0.5$ and $\mu=2$. We observe that the kinetic energy per particle of the solvent component 1 is larger (smaller) than that of the solute component 2 if the former is heavier (ligther) than the latter. The fact that $T_{1} \neq T_{2}$ for arbitrary shear rates indicates that the standard one-temperature Chapman-Enskog theory [2] fails, even for not very disparate masses. In this case, the so-called two-fluid theory must be used [15]. On the other hand, the monotonic behavior for $T_{1} / T_{2}$ found in the steady Couette flow contrasts with the one observed in the uniform shear flow [11]. In the latter problem, for given values of the parameters of the mixture, there exists a particular nonzero value of the shear rate $a$ such that $T_{1}=T_{2}$.

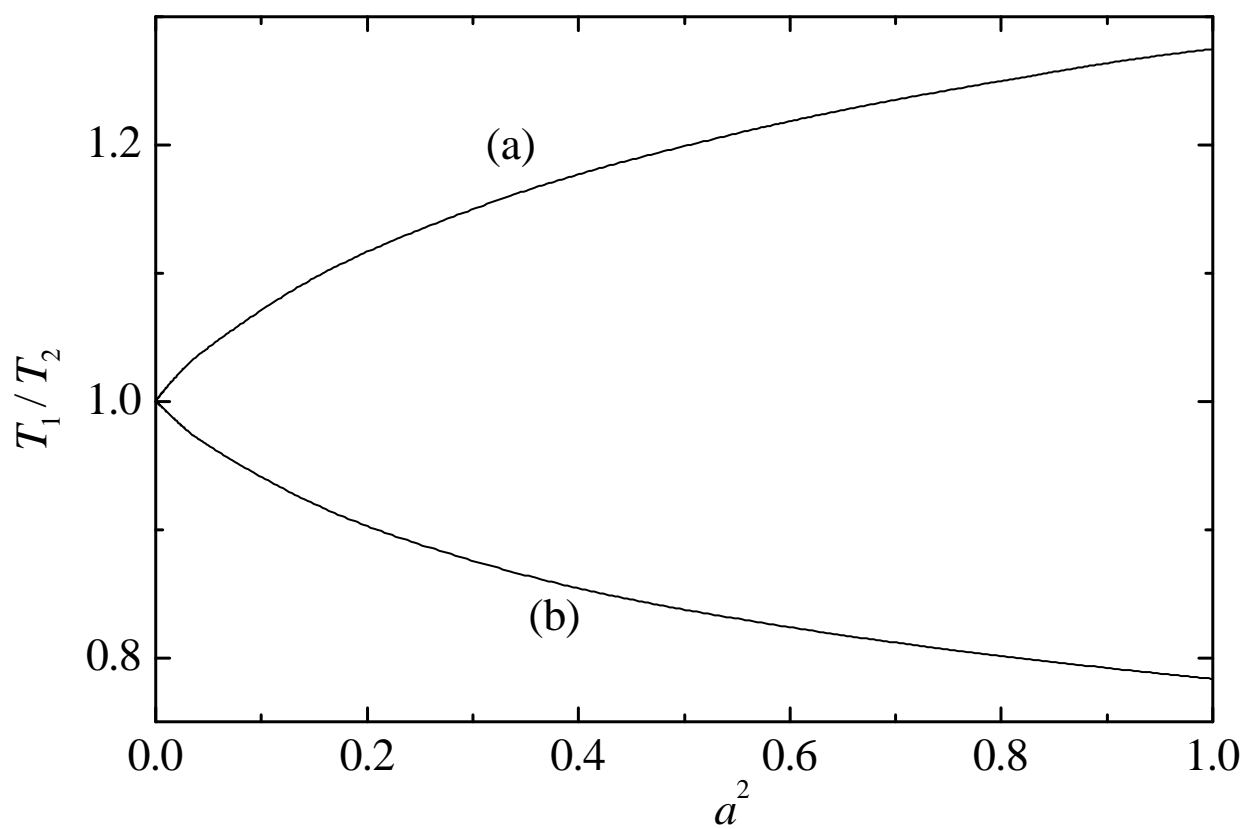

FIG. 1. Shear-rate dependence of the temperature ratio $T_{1} / T_{2}$ for $w_{11}=w_{22}=1, \delta=2$, and two values of the mass ratio $\mu$ : (a) $\mu=2$, and (b) $\mu=0.5$.

The viscosity function $F_{\eta}$ of a binary mixture under Couette flow is defined by Eq. (42). For small shear rates this function behaves as $F_{\eta} \rightarrow 1+F_{\eta}^{(0)} a^{2}$, where

$$
\begin{aligned}
F_{\eta}^{(0)}= & \left(\frac{x_{1}}{\nu_{1}}+\frac{x_{2}}{\nu_{2}}\right)^{-1}\left\{\frac{x_{1}}{\nu_{1}}\left[M \chi_{1}^{(0)}\left(\frac{1}{M}-2 \frac{\nu_{12}}{\nu_{1}}(1+\delta)\right)-18 \gamma_{1}^{(0)}\right]\right. \\
& \left.+\frac{x_{2}}{\nu_{2}}\left[M \chi_{1}^{(0)}\left(2 \frac{\nu_{21}}{\nu_{2}}(1+\delta)-\frac{\delta}{M}\right)-18 \frac{\mu \nu_{1}^{2}}{\nu_{2}^{2}} \gamma_{1}^{(0)}\right]\right\},
\end{aligned}
$$

with $M \equiv \mu /(1+\mu)^{2}$. Depending on the values of the parameters of the mixture, the super-Burnett contribution $F_{\eta}^{(0)}$ can be positive or negative, so for small rates the viscosity function can increase or decrease as the shear rate increases. Nevertheless, outside this small region, $F_{\eta}$ monotonically decreases as the shear rate increases, regardless of the values of the parameters of the mixture as shown in Fig. 2. 


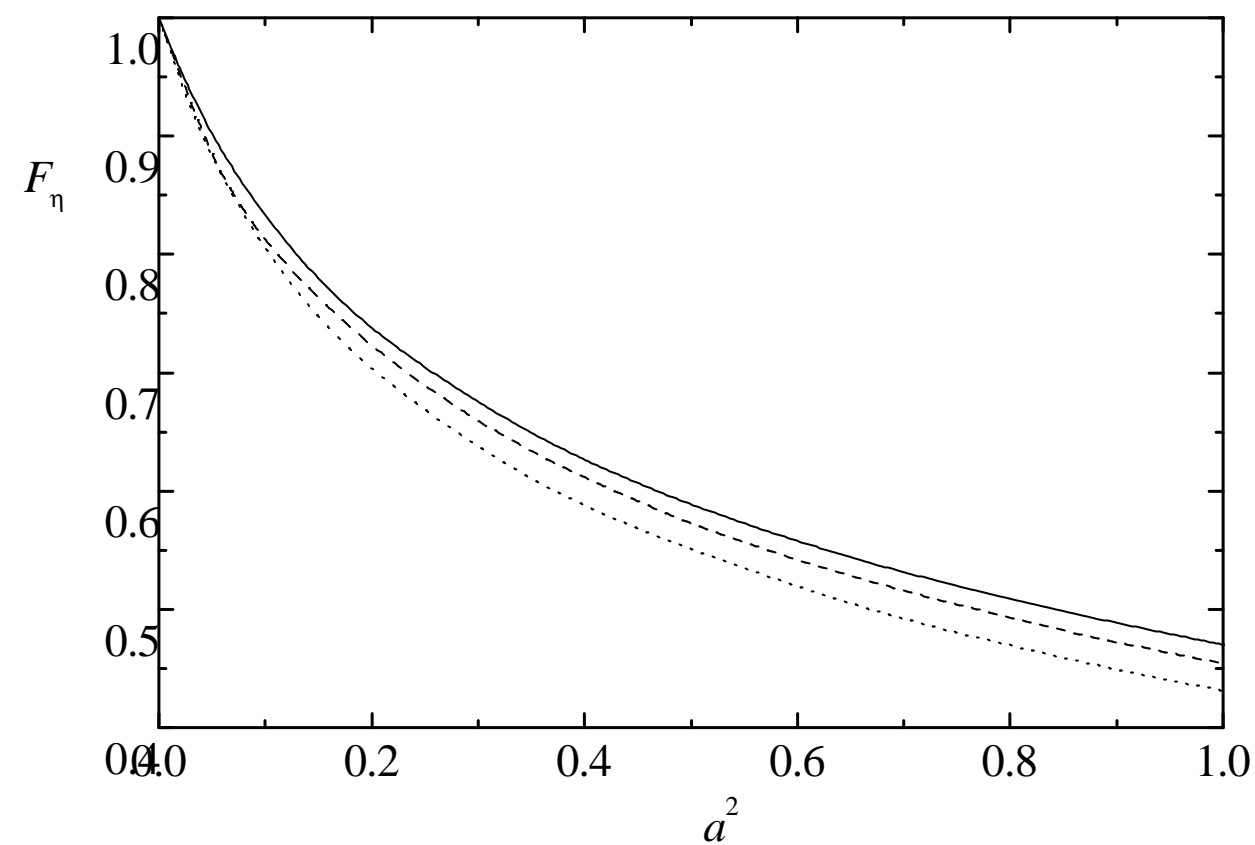

FIG. 2. Shear-rate dependence of the viscosity function $F_{\eta}$ for $w_{11}=w_{22}=1, \delta=2$, and three values of the mass ratio $\mu$ : $\mu=0.5$ (solid line), $\mu=1$ (dashed line), and $\mu=2$ (dotted line).

In addition, at a given value of the shear rate, the viscosity function increases as the mass ratio $m_{1} / m_{2}$ decreases when $n_{1} / n_{2}>1$. The viscometric functions $\Psi_{1,2}$ are given by Eqs. (45) and (46), respectively. In the limit $a \rightarrow 0$, one gets

$$
\begin{gathered}
\Psi_{1}(0)=-14\left(x_{1}+x_{2} \mu \frac{\nu_{1}^{2}}{\nu_{2}^{2}}\right) \gamma_{1}^{(0)} \\
\Psi_{2}(0)=4\left(x_{1}+x_{2} \mu \frac{\nu_{1}^{2}}{\nu_{2}^{2}}\right) \gamma_{1}^{(0)} .
\end{gathered}
$$

Notice that $\Psi_{1,2}(0)$ are Burnett transport coefficients. The shear rate dependence of the (dimensionless) viscometric functions $\Psi_{1,2}(a)$ is shown in Figs. 3 and 4 , respectively, for the same cases as in Fig. 2. The first viscometric function is always negative while the second one is positive. In general, the magnitude of both viscometric functions decreases with the shear rate. On the other hand, for zero shear rates, $\left|\Psi_{1,2}(0)\right|_{\mu=0.5}<\left|\Psi_{1,2}(0)\right|_{\mu=1}<\left|\Psi_{1,2}(0)\right|_{\mu=2}$. These inequalities are kept for finite shear rates. 


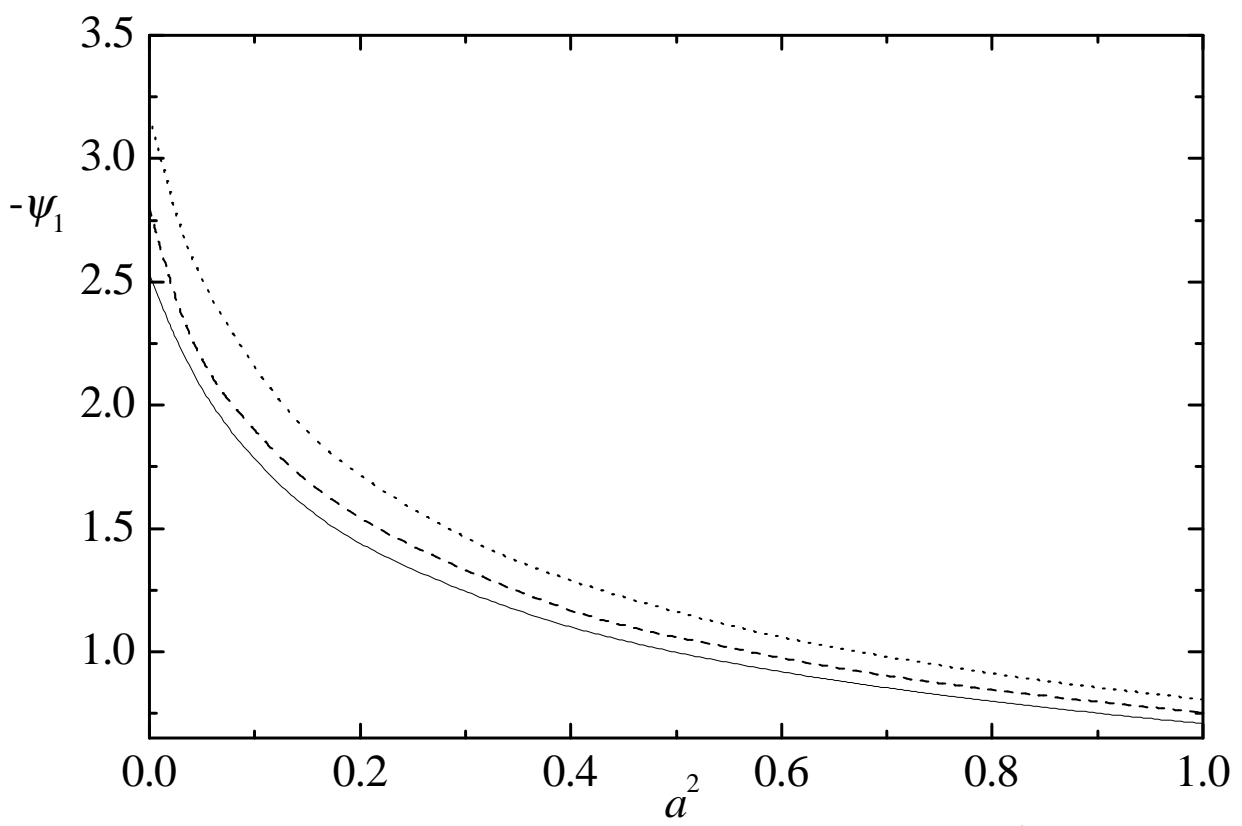

FIG. 3. Shear-rate dependence of the first viscometric function $-\Psi_{1}$ for $w_{11}=w_{22}=1, \delta=2$, and three values of the mass ratio $\mu: \mu=0.5$ (solid line), $\mu=1$ (dashed line), and $\mu=2$ (dotted line).

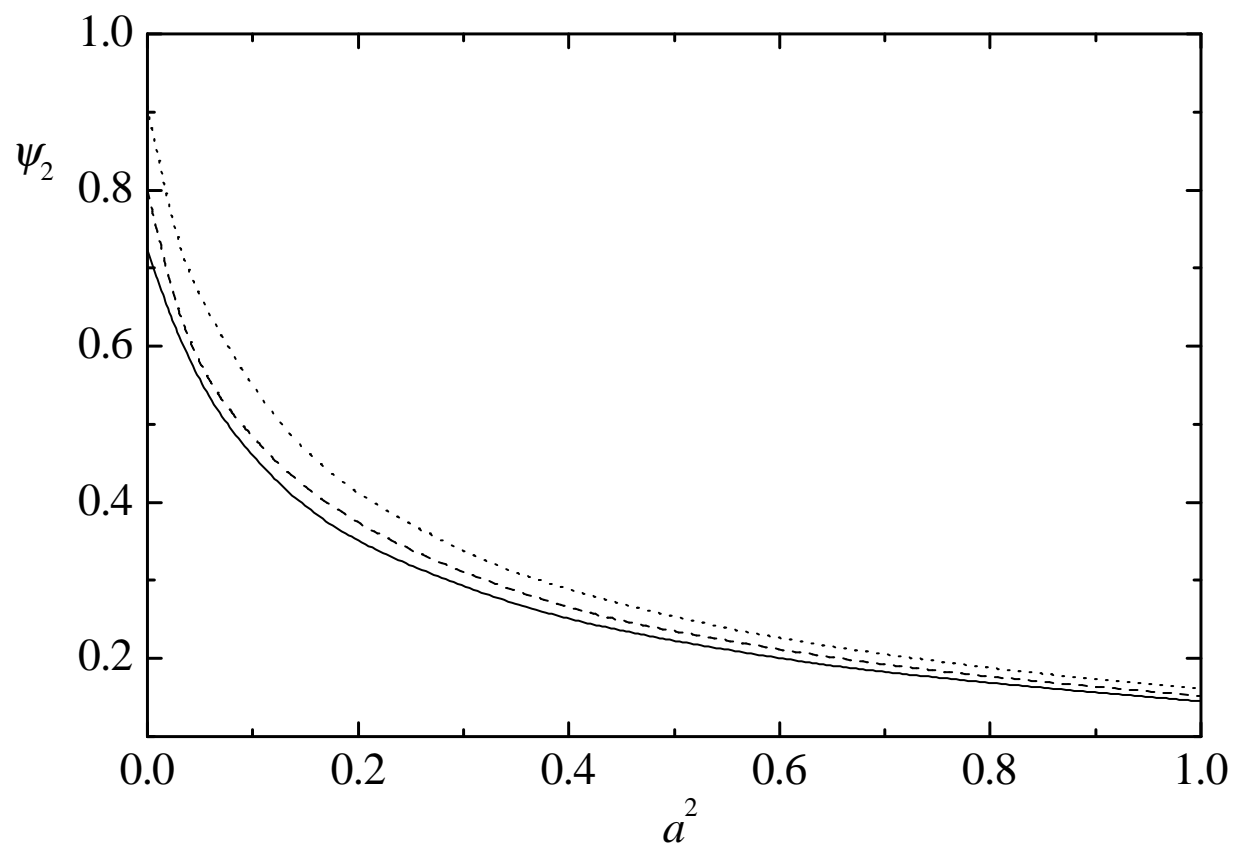

FIG. 4. Shear-rate dependence of the second viscometric function $\Psi_{2}$ for $w_{11}=w_{22}=1, \delta=2$, and three values of the mass ratio $\mu: \mu=0.5$ (solid line), $\mu=1$ (dashed line), and $\mu=2$ (dotted line).

The transport of energy is characterized by the coefficients $F_{\lambda}$ and $\Phi$. For small shear rates, $F_{\lambda} \rightarrow 1+F_{\lambda}^{(0)} a^{2}$ and $\Phi \rightarrow \Phi(0)$, with

$$
\begin{aligned}
F_{\lambda}^{(0)}= & \left(\frac{x_{1}}{m_{1} \nu_{1}}+\frac{x_{2}}{m_{2} \nu_{2}}\right)^{-1}\left\{\frac{x_{1}}{m_{1} \nu_{1}}\left[2 M \chi_{1}^{(0)}\left(\frac{1}{M}-\frac{\nu_{12}}{\nu_{1}}(1+\delta)\right)-\frac{252}{5} \gamma_{1}^{(0)}-\frac{36}{5} \frac{\nu^{2}}{\nu_{1}^{2}}\right]\right. \\
& \left.+\frac{x_{2}}{m_{2} \nu_{2}}\left[2 M \chi_{1}^{(0)}\left(\frac{\nu_{21}}{\nu_{2}}(1+\delta)-\frac{\delta}{M}\right)-\frac{252}{5} \frac{\mu \nu_{1}^{2}}{\nu_{2}^{2}} \gamma_{1}^{(0)}-\frac{36}{5} \frac{\nu^{2}}{\nu_{2}^{2}}\right]\right\} .
\end{aligned}
$$




$$
\Phi(0)=-\frac{14}{5} \frac{\nu}{\nu_{1}} \frac{\delta+\mu\left(\nu_{1} / \nu_{2}\right)^{2}}{\delta+\mu\left(\nu_{1} / \nu_{2}\right)}
$$

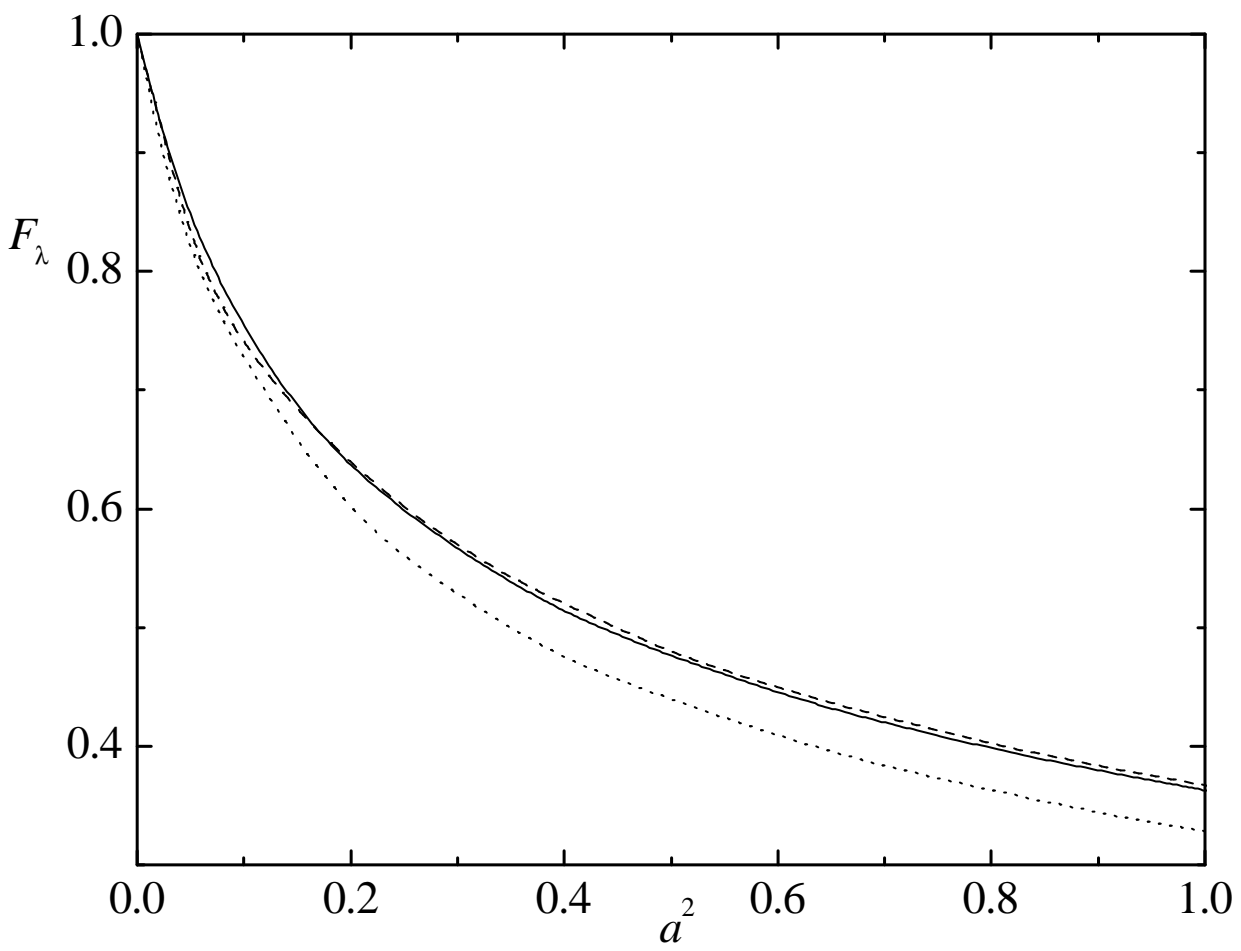

FIG. 5. Shear-rate dependence of the generalized thermal conductivity function $F_{\lambda}$ for $w_{11}=w_{22}=1, \delta=2$, and three values of the mass ratio $\mu: \mu=0.5$ (solid line), $\mu=1$ (dashed line), and $\mu=2$ (dotted line).

It is apparent that the coefficient $F_{\lambda}^{(0)}$ presents a complex dependence on the parameters of the mixture. In general, and in accordance with Fig. 5 , for finite values of the shear rate the generalized thermal conductivity coefficient $F_{\lambda}$ decreases as the shear rate increases with independence of the values of the parameters of the mixture. It is interesting to remark that the curves of $\mu=0.5$ and $\mu=1$ are practically the same in the range of shear rates considered. In Fig. 6 we plot the shear rate dependence of the reduced coefficient $\Phi(a)$. We observe that $\Phi$ is negative and its magnitude decreases with the shear rate. Further, in the range of shear rates studied, this coefficient is practically independent of the mass ratio. This is rather surprising if one takes into account that this is a coefficient measuring complex coupling effects between both gradients and a more significant influence of the parameters of the mixture should be expected. 


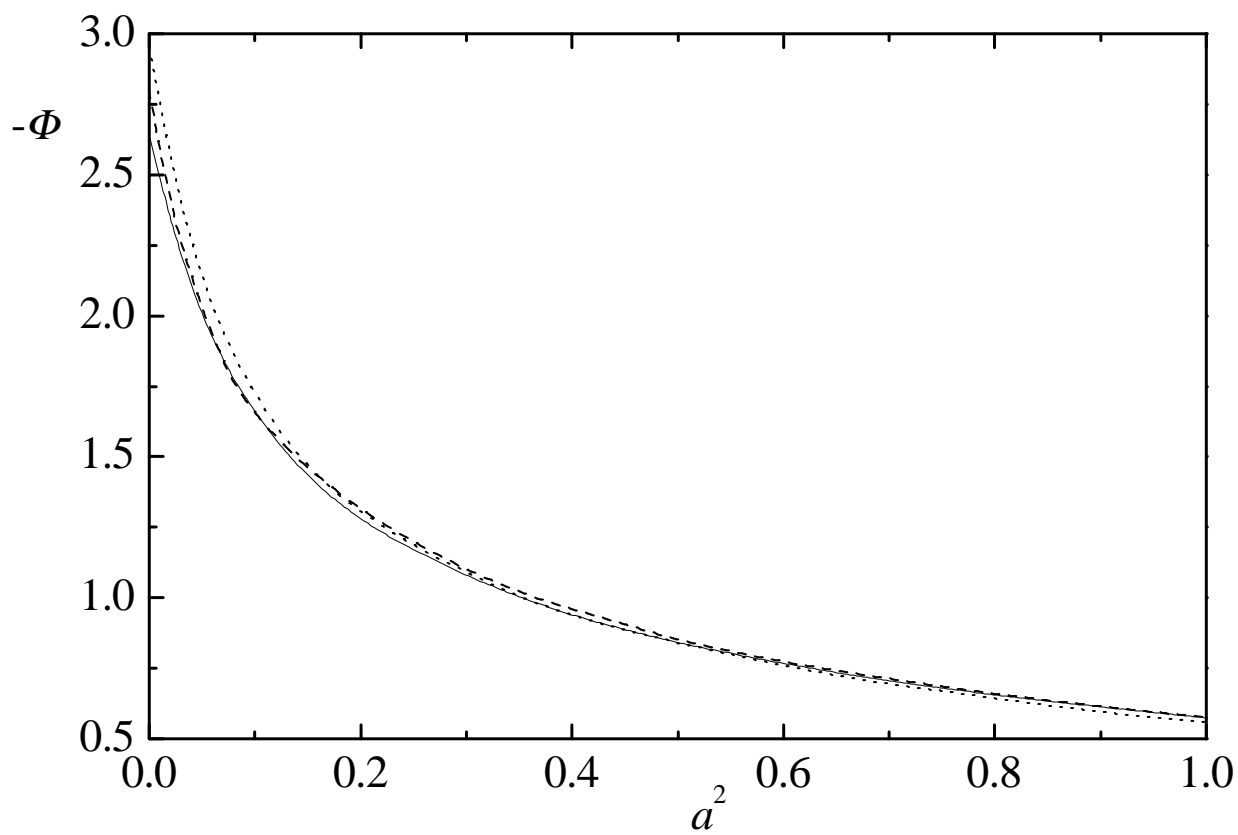

FIG. 6. Shear-rate dependence of the cross coefficient $-\Phi$ for $w_{11}=w_{22}=1, \delta=2$, and three values of the mass ratio $\mu$ : $\mu=0.5$ (solid line), $\mu=1$ (dashed line), and $\mu=2$ (dotted line).

Finally, let us note that in the tracer limit $\left(n_{1} / n_{2} \rightarrow 0\right)$, all the results of the binary mixture reduce to those previously obtained from the GK model [9].

\section{DISCUSSION}

In this paper we have considered a gas mixture of $N$ species in a steady nonequilibrium state involving combined heat and momentum transport. This state is usually referred to as the steady planar Couette flow. The system is a gas mixture enclosed between two relatively moving parallel plates which are kept in general at different temperatures. The shear rate and the thermal gradient are the two nonequilibrium parameters of the problem. Under these conditions, the density, flow velocity, and the temperature are nonhomogeneous, thus making the analysis of this problem more complicated than in other nonequilibrium situations. Further, the mixture is not restricted to specific values of the mass ratios, concentration ratios and size ratios. As a consequence, the transport properties of the system are nonlinear functions of the strengths of the gradients and of the parameters of the mixture. In order to offer a description as detailed as possible, we have used a kinetic model of the Boltzmann equation. Specifically, we have considered the GK model for mixtures, whose reliability has been proved in the past in several nonequilibrium problems [16,17]. The explicit determination of the transport properties of the gas from an exact solution of the GK model has been the goal of this paper. It is important to remark that progress has been possible here due to the previous results derived in the limit cases of mechanically equivalent particles $\sqrt{7]}$ and tracer particles [9].

Guided by the results obtained for a single gas [- [], we have found a consistent solution of the GK equation characterized by a uniform presure, and linear velocity and parabolic temperature profiles with respect to a certain scaled variable (distance times the local collision frequency). The consistency condition for the partial temperatures $T_{i}$ leads to a set of $N$ coupled transcendent equations, whose (numerical) solution gives $T_{i}$ in terms of the shear rate and the parameters of the mixture. Once the $T_{i}$ 's are known, we explicitly evaluate the pressure tensor and the heat flux. The nonzero elements of the pressure tensor define three relevant transport coefficients: the generalized shear viscosity $F_{\eta}$, Eqs. (40) and (42), the first viscometric function $\Psi_{1}$, Eqs. (43) and (45), and the second viscometric function $\Psi_{2}$, Eqs. (44) and (46). While the viscosity function measures shear stresses, the two viscometric functions provide information on normal stresses. The heat flux turns out to be exactly proportional to the thermal gradient, with independence of its magnitude, but it defines two shear rate dependent transport coefficients: the generalized thermal conductivity $F_{\lambda}$, Eqs. (47) and (50), and a generalized cross coefficient $\Phi$, Eqs. (48) and (51), which accounts for the transport of energy along the direction normal to the thermal gradient. For the sake of illustration, a binary mixture has been considered. The results show that, except perhaps in a narrow region small of shear rates, the magnitude of these coefficients decreases for finite shear fields. Consequently, the main effect of the shear flow is to 
inhibit the transport of momentum and energy across the system. In general, this inhibition is more significant when the excess component is heavier than the defect component in the case of the coefficients $F_{\eta}$ and $F_{\lambda}$, while the opposite happens in the case of the two viscometric functions. Concerning the cross coefficient $\Psi$ (which is negative), the results indicate that in the range of shear rates considered is practically independent of the values of the parameters of the mixture.

The problem studied here is worthwhile by itself. Furthermore, it can be taken as a starting point to analyze mutual diffusion under steady Couette flow. In the same way as done in the tracer limit [18, 19, the idea is to carry out a perturbation expansion in powers of the molar fraction gradient around the solution found in this paper. In the first order of the expansion, a generalized Fick's law is expected, where a shear rate dependent mutual diffusion tensor can be identified. On the other hand, it is apparent that the results reported here can also be of relevance in connection with computer simulations. In the context of molecular dynamics simulations, it is difficult to achieve large shear rates in the bulk region to clearly observe nonlinear effects in the transport of momentum and energy. One possibility to overcome such problems inherent to molecular dynamics in the low-density regime is to use the direct simulation Monte Carlo method [20], which has been shown to be fruitful in several problems. We hope that the results derived here for the nonlinear transport coefficients may stimulate the performance of computer simulations to check the reliability of our predictions. Recent comparison made for a single gas under Couette flow supports the accuracy of the BGK results.

\section{ACKNOWLEDGMENTS}

I am grateful to Dr. A. Santos for a critical reading of the manuscript. Partial support from the DGES (Spain) through grant No. PB97-1501 and from the Junta of Extremadura (Fondo Social Europeo) through grant No. IPR98C019 is acknowledged.

\section{APPENDIX A: VELOCITY MOMENTS OF THE DISTRIBUTION FUNCTIONS}

In this Appendix we evaluate the velocity integrals for the consistency conditions and for the momentum and heat

fluxes. They correspond to the low order moments of the distribution functions $f_{i}$. Let us consider the formal solution to Eq. (18) given by

$$
\begin{aligned}
f_{i} & =\left(1+\frac{v_{y}}{\nu_{i}} \frac{\partial}{\partial y}\right)^{-1} \sum_{j=1}^{N} \frac{\nu_{i j}}{\nu_{i}} f_{i j} \\
& =\sum_{j=1}^{N} \frac{\nu_{i j}}{\nu_{i}} \Lambda_{i j}
\end{aligned}
$$

where

$$
\begin{aligned}
\Lambda_{i j} & =\left(1+\frac{v_{y}}{\nu_{i}} \frac{\partial}{\partial y}\right)^{-1} f_{i j} \\
& =\sum_{k=0}^{\infty}\left(-v_{y}\right)^{k}\left(\frac{1}{\nu_{i}} \frac{\partial}{\partial y}\right)^{k} f_{i j} .
\end{aligned}
$$

We define the velocity integrals

$$
M_{k_{1}, k_{2}, k_{3}}^{i j}=\int d \mathbf{v} V_{x}^{k_{1}} V_{y}^{k_{2}} V_{z}^{k_{3}} \Lambda_{i j}
$$

From a formal point of view, the moments defined in Eq. A3 can be directly obtained from the results derived in the one-component case [7, 14, when one exploits the equivalence between the hydrodynamic profiles (20)-(22) with those given in the single gas. Consequently, the moments (A3) can be determined from comparison with those of the distribution function $f$ of the one-component gas by making the changes $\nu \rightarrow \nu_{i j}, \nu^{-1} \partial_{y} u_{x} \rightarrow a_{i}, T \rightarrow T_{i j}$, and $\left(\nu^{-1} \partial_{y}\right)^{2} T=-\left(2 m / k_{B}\right) \gamma \rightarrow-\left(2 m_{i} / k_{B}\right) \gamma_{i j}$. Taking into account this equivalence, we can explicitly write the first few moments of $\Lambda_{i j}$. Here, we display the moments with degree $k_{1}+k_{2}+k_{3} \leq 3$ appearing in the calculations performed along the main text. They are given by $[7,14$ 


$$
\begin{gathered}
M_{0,0,0}^{i j}=n_{i}, \\
M_{1,0,0}^{i j}=M_{0,1,0}^{i j}=M_{0,0,1}^{i j}=0, \\
M_{2,0,0}^{i j}=\frac{n_{i} k_{B} T_{i j}}{m_{i}}\left[1+4 \gamma_{i j}\left(F_{1}+F_{2}\right)\right], \\
M_{0,2,0}^{i j}=\frac{n_{i} k_{B} T_{i j}}{m_{i}}\left[1-2 \gamma_{i j}\left(F_{1}+2 F_{2}\right)\right], \\
M_{0,0,2}^{i j}=\frac{n_{i} k_{B} T_{i j}}{m_{i}}\left(1-2 \gamma_{i j} F_{1}\right), \\
M_{1,1,0}^{i j}=-\frac{n_{i} k_{B} T_{i j}}{m_{i}} F_{0} a_{i}, \\
M_{3,0,0}^{i j}=2 \frac{n_{i} k_{B}^{2} T_{i j}}{m_{i}^{2} \nu_{i}}\left[3 F_{2}+2 a_{i}^{2}\left(4 F_{5}+8 F_{4}+5 F_{3}+F_{2}\right)\right] a_{i} \frac{\partial}{\partial y} T_{i j} . \\
M_{1,0,2}^{i j}=2 \frac{n_{i} k_{B}^{2} T_{i j}}{m_{i}^{2} \nu_{i}} F_{1} \frac{\partial}{\partial y} T_{i j}, \\
m_{i}^{2} \nu_{i} \\
M_{0,3,0}^{i j}=-\frac{n_{i} k_{B}^{2} T_{i j}}{m_{i}^{2} \nu_{i}}\left(2 F_{2}+F_{1}\right) \frac{\partial}{\partial y} T_{i j}, \\
M_{1,2,0}^{i j}=2 \frac{n_{i} k_{B}^{2} T_{i j}}{m_{i}^{2} \nu_{i}}\left(2 F_{3}+F_{2}\right) a_{i} \frac{\partial}{\partial y} T_{i j},
\end{gathered}
$$

In these expressions, $\gamma_{i j}=\chi_{i j} \gamma_{i}, \chi_{i j}$ is defined in Eq. 28) and

$$
F_{r} \equiv F_{r}\left(\gamma_{i j}\right)=\left(\frac{d}{d \gamma_{i j}} \gamma_{i j}\right)^{r} F_{0}\left(\gamma_{i j}\right)
$$

with $F_{0}\left(\gamma_{i j}\right)$ given by Eq. (30).

The consistency conditions for the density and the flow velocity are easily seen to be verified by using Eqs. (A4) and (A5), respectively. The condition for the temperature on the other hand requires that

$$
\sum_{j=1}^{N} \frac{\nu_{i j}}{\nu_{i}}\left(M_{2,0,0}^{i j}+M_{0,2,0}^{i j}+M_{0,0,2}^{i j}\right)=3 \frac{n_{i} k_{B} T_{i}}{m_{i}} .
$$

Substitution of Eqs. ( $\mathrm{A} 6)-(\mathrm{A} 8)$ into Eqs. (A17) yields the set of coupled equations $(27)$. The momentum and heat fluxes can be evaluated from the remaining velocity moments. The pressure tensor can be written as 


$$
\mathrm{P}=\sum_{i=1}^{N} \int d \mathbf{v} m_{i} \mathbf{V} \mathbf{V} f_{i}=\sum_{i=1}^{N} \mathrm{P}_{i},
$$

where the nonzero elements of $\mathrm{P}_{i}$ are given by

$$
\left\{P_{i, x x} ; P_{i, y y} ; P_{i, z z} ; P_{i, x y}\right\}=\sum_{j=1}^{N} m_{i} \frac{\nu_{i j}}{\nu_{i}}\left\{M_{2,0,0}^{i j} ; M_{0,2,0}^{i j} ; M_{0,0,2}^{i j} ; M_{1,1,0}^{i j}\right\} .
$$

The heat flux can be obtained in a similar way. It can be also written as

$$
\mathbf{q}=\sum_{i=1}^{N} \int d \mathbf{v} \frac{m_{i}}{2} V^{2} \mathbf{V} f_{i}=\sum_{i=1}^{N} \mathbf{q}_{i}
$$

where

$$
\begin{aligned}
q_{y, i} & =\sum_{j=1}^{N} \frac{m_{i}}{2} \frac{\nu_{i j}}{\nu_{i}}\left(M_{2,1,0}^{i j}+M_{0,3,0}^{i j}+M_{0,1,2}^{i j}\right), \\
q_{x, i} & =\sum_{j=1}^{N} \frac{m_{i}}{2} \frac{\nu_{i j}}{\nu_{i}}\left(M_{3,0,0}^{i j}+M_{1,2,0}^{i j}+M_{1,0,2}^{i j}\right) .
\end{aligned}
$$

The explicit expressions for the fluxes and transport coefficients appearing in the text can be easily derived from Eqs. (A18)-(A22).

[1] J. A. McLennan, Introduction to Nonequilibrium Statistical Mechanics, Prentice Hall, Englewood Cliffs, NJ, 1989.

[2] S. Chapman, T. G. Cowling, The Mathematical Theory of Nonuniform Gases, Cambridge University Press, Cambridge, 1970.

[3] M. Tij, A. Santos, Phys. Fluids 7 (1995) 2858.

[4] D. Risso, P. Cordero, Phys. Rev. E 56 (1997) 489.

[5] E. P. Gross, M. Krook, Phys. Rev. 102 (1956) 593.

[6] P.L. Bhatnagar, E. P. Gross, M. Krook, Phys. Rev. 94 (1954) 511.

[7] J. J. Brey, A. Santos, J. W. Dufty, Phys. Rev. A 36 (1987) 2842.

[8] C. S. Kim, J. W. Dufty, A. Santos, J. J. Brey, Phys. Rev. A 40 (1989) 7165.

[9] V. Garzó and A. Santos, Phys. Rev. E 48 (1993) 256.

[10] C. Marín, V. Garzó, A. Santos, J. Stat. Phys. 75 (1994) 797.

[11] C. Marín, V. Garzó, A. Santos, Phys. Rev. E 52 (1995) 3812.

[12] C. Marín, J. J. Montanero, V. Garzó, Physica A 225 (1996) 235.

[13] M. Abramowitz, I. A. Stegun, Handbook of Mathematical Functions, Dover, New York, 1972.

[14] J. M. Montanero, V. Garzó, Phys. Rev. E 58 (1998) 1836.

[15] C. J. Goebel, S. M. Harris, E. A. Johnson, Phys. Fluids 19 (1976) 627.

[16] C. Marín, J. M. Montanero, V. Garzó, Mol. Phys. 88 (1996) 1249.

[17] V. Garzó, C. Marín, in: S. G. Pandalai (Ed.), Recent Research Developments in Physics of Fluids 1, Transworld Research Network, Trivandrum, 1998.

[18] V. Garzó, A. Santos, Phys. Rev. E 52 (1995) 4942.

[19] V. Garzó, Physica A 234 (1996) 108.

[20] G. A. Bird, Molecular Gas Dynamics and the Direct Simulation Monte Carlo of Gas Flows, Clarendon, Oxford, 1994. 